\title{
EDITORIAL
}

\section{Training Control And Performance Assessment In Sport}

\author{
Ricardo J. Fernandes ${ }^{1,2^{*}}$, Júlio Garganta ${ }^{1}$ and Maria Teresa Anguera ${ }^{3}$ \\ ${ }^{I}$ Centre of Research, Education, Innovation and Intervention in Sport (CIFI2D), Faculty of Sport, University of Porto, \\ Porto, Portugal \\ ${ }^{2}$ Porto Biomechanics Laboratory (LABIOMEP), University of Porto, Porto, Portugal \\ ${ }^{3}$ Institute for Research on the Brain, Cognition and Behaviour, IR3C, Faculty of Psychology, University of Barcelona, \\ Barcelona, Spain
}

In sport contexts, performance is developed through a training process involving a suitable combination of several variables. This is a complex process, in which certain features are mixed and sequenced so when the athletes compete they will have maximized their skills for optimal performance. Thus, training should be regarded as a multifaceted practice, in which the inter-relationships between the different features complement its diachronic and synchronic characteristics.

This complementarity involves areas such as physiology, biomechanics, decision making and match analysis, and each one entails a range of constraints that determine the level of performance that an athlete or a team will ultimately reach. In terms of physiology, which concerns the set of vital parameters required for the physical functioning of the human body, aspects such as speed, oxygen consumption and blood lactate concentrations are all known to be relevant. Regarding biomechanics, it is necessary to consider technical effectiveness, high-level routines and the actual methods that are required, whether in general or among specific groups, such as young people. Another relevant facet of training is decision making, being particularly relevant the central role played by perceptual/cognitive aspects and procedural skills. Concerning match analysis, it involves a wide range of issues including the analysis of goal scoring, the practical implications of task constraints, the influence of effective time, or the role played by contextual variables. In the studies featured in this special issue the reader will find these aspects addressed by specialists in the field.

In this sense, the comprehensive monitoring of athletes is necessary at both the preparatory and competitive stages, allowing coaches to make informed decisions regarding the effects of planning and training. Hence, collaborations

\footnotetext{
*Address correspondence to this author at the Centre of Research, Education, Innovation and Intervention in Sport (CIFI2D), Faculty of Sport, University of Porto, Porto, Portugal; Tel: +351225074763;

Fax: +35155000687; E-mail: ricfer@fade.up.pt
}

between coaches and researchers often involve the evaluation and control of training programmes, since it is important to identify athletes' strengths and weaknesses. Furthermore, because both are interested in discovering the most effective ways of improving performance, it is crucial to capture, analyse and evaluate information on key features, such as the physical, mental, technical and tactical skills of athletes. This is why scientific techniques and strategies to enhance sport performance have been progressively incorporated into coaching practices and research programmes.

The literature shows how scientific information and knowledge can be an effective way of improving performance in sport, providing a relevant framework for the development of research and the optimization of sports practice in different settings. This special issue pays particular attention to the approaches, constraints and methodological possibilities that characterize sports sciences. In this regard, it is important to recognize that the implementation of different methodologies constitutes a dynamic aspect that evolves and assumes particular relevance at different points of the research process. Indeed, it encompasses the initial proposal of the study design, which will serve as the core of the process, the choice of variables that need to be considered. In this regard, it should be highlighted the significant recent developments in data capture software and systems for monitoring data quality, as well as the wide range of techniques that are now available. This has enabled results to be generalized to new contexts and to different sports. It is clear, therefore, that the complexity mentioned at the outset affects both the conceptual and the methodological domains.

This special issue of the OSSJ, entitled Training Control and Performance Assessment in Sport, offers a collection of papers focusing on physiology, biomechanics, decision making and match analysis. Some studies arises in the scope of a particular project granted by the Portuguese Agency for Science and Technology (PTDC/DES/101224/2008- 
FCOMP-01-0124-FEDER-009577) We hope that readers will find the content to be of interest, and that it encourages deeper and more active reflection among researchers, coaches, sportsmen and women, managers and all those who, through their daily work, contribute to the improvement of sport performance.

(C) Fernandes et al.; Licensee Bentham Open.

This is an open access article licensed under the terms of the Creative Commons Attribution Non-Commercial License (http://creativecommons.org/licenses/ by-nc/3.0/) which permits unrestricted, non-commercial use, distribution and reproduction in any medium, provided the work is properly cited. 